\title{
Fotopolimerização: Princípios e Métodos
}

\author{
Máira R. Rodrigues \\ Instituto de Pesquisa e Desenvolvimento, Universidade do Vale do Paraíba
}

\author{
Miguel G. Neumann \\ Instituto de Química de São Carlos, USP
}

Resumo: O interesse na Fotoquímica de Polímeros tem crescido nas últimas décadas devido não somente ao grande número de novas aplicações como também à repercussão do ponto de vista econômico, técnico e ecológico. A fotopolimerização/fotocura tem recebido a atenção especial devido às incontáveis aplicações e a sua importância na área de Materiais. A cinética destes processos necessita de métodos analíticos precisos e rápidos para ser avaliada. Entre os métodos analíticos convencionais, os mais usados são a gravimetria e a medida de propriedades macroscópicas, como resistência à tração, à deformação e dureza. Entretanto, para determinações mais analíticas é conveniente usar métodos que permitam estudar a cinética de fotopolimerização em tempo real. Neste sentido têm sido usadas técnicas como dilatometria, espectroscopia Raman, calorimetria, e espectroscopia de infravermelho por transformada de Fourier em tempo real. A atividade no campo da polimerização continua a se expandir em muitas áreas e, recentemente, o interesse nos sistemas do fotoiniciação envolvendo a polimerização via radicais livres, iniciada por luz visível, tem recebido atenção especial. Recentemente, estudos sobre fotopolimerização catiônica induzida pela luz visível começaram a ser pesquisados novamente devido à grande variedade de monômeros que são polimerizáveis por via catiônica mas não por via radicar, como, por exemplo, oxiranas e éteres vinílicos.

Palavras-chave: Fotopolimerização catiônica, fotopolimerização via radicalar, dilatometria, calorimetria, gravimetria, espectroscopia de infravermelho por transformada de Fourier.

\section{Photopolymerization: Principles and Methods}

Abstract: The interest for Polymer Photochemistry has been growing in the last decades as a consequence not only of the great number of new applications as well as due to the repercussion of economical, technical and ecological characters. The photopolymerization - photocuring processes have received special attention due to the countless and important applications in the area of Materials. The kinetics of these processes needs fast and precise analytic methods to be evaluated. Among the conventional analytic methods, the most used are gravimetry and the determination of macroscopic properties, as resistance to traction, deformation and hardness. However, for a better insight in the reactions it is convenient to use more advanced analytic methods, in order to study the photopolymerization kinetics in real time. Dilatometry, Raman spectroscopy, calorimetry, and real time Fourier transform infrared spectroscopy are some of the techniques that have been used. Activity in the field of photopolymerization continues to expand in many areas and recently, the interest in photoinitiation systems involving the initiation of free-radical polymerization with visible light irradiation has been retaken. Additionally, studies on cationic photopolymerization induced by visible light started again to be studied because of the large variety of monomers that can be photopolymerized by via cation initiation, but not by free radicals, such as oxiranes and vinyl ethers.

Keywords: Cationic photopolymerization, free-radical photopolymerization, dilatometry, calorimetry, gravimetry, Fourier transform infrared spectroscopy.

\section{Introdução}

Atualmente a Fotoquímica constitui uma área de pesquisa multidisciplinar que está tendo um grande desenvolvimento tanto em nível acadêmico como industrial. Entretanto, devido à dificuldade inerente à incorporação de luz artificial a um processo industrial há uma defasagem entre os aspectos básicos e aplicados da Fotoquímica $^{[1,2]}$. A Fotoquímica de Polímeros é uma das áreas que mais tem se desenvolvido em nível industrial, devido não só ao grande número de novas aplicações como também do ponto de vista econômico, técnico e ecológico. Em nível industrial, a busca de sistemas livres de solventes orgânicos, de baixo custo energético e de aplicação rápida mesmo a temperatura ambiente tem motivado a incorporação de processos baseados na Fotoquímica de Polímeros.

As aplicações da Fotoquímica de Polímeros podem ser divididas em três grandes grupos: Reações de Síntese ${ }^{[3,4]}$,

Autor para correspondência: Miguel G. Neumann, USP, Instituto de Química de São Carlos, Caixa Postal 780, CEP:13560-970, São Carlos, SP, E-mail: neumann@iqsc.sc.usp.br 
Fotodegradação/Fotoestabilização ${ }^{[5,6]}$ e processos de Fotopolimerização/Fotocura ${ }^{[7,8]}$.

O setor de fotopolimerização/fotocura tem recebido atenção especial devido a inúmeras e importantes aplicações na área de Materiais, como por exemplo:

- Odontologia ${ }^{[9,10]}$. As resinas fotopolimerizáveis para restaurações foram desenvolvidas com a finalidade de suprir a principal desvantagem dos sistemas de polimerização quimicamente induzidos: a falta de controle sobre o tempo de reação. As resinas ativadas por luz visível iniciam o processo de polimerização através da absorção de luz de um iniciador (geralmente uma $\alpha$-dicetona) que uma vez ativado reage com um agente redutor (amina alifática) para produzir radicais livres. A partir daí ocorre à polimerização dos monômeros metacrílicos que formam uma matriz polimérica com ligações cruzadas.

- Óptica ${ }^{[10,11]}$. Vários tipos de lentes de contato envolvem materiais poliméricos como poli(metacrilato de metila), poli(metacrilato de hidroxietila) ou copolímeros destes com vinilpirrolidona, produzidos por polimerização térmica ou fotoiniciada.

- Artes Gráficas. Uso de tintas, vernizes e adesivos de fotossecagem rápida ${ }^{[10,12]}$.

- Eletricidade e Eletrônica. O exemplo mais conhecido é a fabricação de circuitos impressos, embora outras aplicações como a fotoestereolitografia estejam sendo cada vez mais usadas ${ }^{[13,14]}$.

Do ponto de vista formal e considerando o mecanismo intrínseco da fotoformação do polímeros, a luz interage na etapa de iniciação da polimerização ou entrecruzamento, sendo portanto a etapa de fotoiniciação o ponto central de interesse fotoquímico.

Os sistemas fotopolimerizáveis envolvem modificações das formulações usadas para produzir materiais com propriedades específicas. Por exemplo, na área de recobrimentos os componentes básicos das formulações são: sistema fotoiniciador (1-3\% em peso), oligômeros ( $25-90 \%$ em peso), monômeros (15-60\% em peso) e aditivos ( $1-50 \%$ em peso). Como geralmente os monômeros têm baixo coeficiente de extinção molar é necessário adicionar à formulação um sistema fotoiniciador que absorva luz e produza as espécies reativas, radicais ou íons, que iniciem a polimerização. Os oligômeros fornecem propriedades especiais ao substrato a que se incorporam proporcionando uma ampla variedade de possibilidades no desenvolvimento de resinas para diferentes aplicações. A seleção do monômero é determinada pelos seguintes requisitos: controle de viscosidade, efeito na velocidade de fotopolimerização e nas propriedades do material final, volatilidade, odor e toxidade. Os aditivos modificam as propriedades do material no sentido desejado; entre eles cabe citar cargas, surfactantes, pigmentos, etc ${ }^{[2]}$.

A atual preocupação ecológica potencializa novos procedimentos mais respeitosos com o meio ambiente, favorecendo a utilização de sistemas aquosos. O leque de possibilidades de aplicação e de produção de novos polímeros fez com que a polimerização em meios dispersos tenha sofrido um avanço muito importante do ponto de vista de pesquisa, nos últimos anos. Neste sentido, a fotopolimerização em emulsão também é um processo importante na produção industrial de polímeros em forma de látex, ou colóides para aplicações como adesivos, tintas, esmaltes ou outros recobrimentos ${ }^{[15]}$. A principal vantagem em comparação com outras técnicas de polimerização é a obtenção de altas massas molares a altas velocidades de conversão. Além disso, é uma técnica adequada para a obtenção de materiais compostos nos quais um componente inorgânico é recoberto parcial ou totalmente com um polímero (encapsulamento) ${ }^{[16]} \mathrm{e}$ para a preparação de látex de redes interpenetradas ${ }^{[17]}$. Também a polimerização em biou multicamadas é objeto de crescente interesse como, por exemplo, filmes poliméricos ultrafinos, que podem ser utilizados na produção de sensores bioquímicos ${ }^{[18]}$.

É possível preparar emulsificantes que incluem grupos funcionais polimerizáveis ou unidos ao iniciador ou a agentes de transferência, com o objetivo de unir covalentemente o emulsificante ao polímero. Este procedimento visa a melhora em algumas das propriedades do látex e dos filmes preparados a partir deles ${ }^{[19]}$.

Neste trabalho, pretendemos discorrer sobre parâmetros da fotopolimerização em cadeia via catiônica e via radicalar e exemplificar alguns métodos de determinação da velocidade de fotopolimerização. Embora existam outros métodos pouco ou não citados aqui, a discussão será mais detalhada em estudos já realizados por nós.

\section{Polimerização em cadeia ${ }^{[20]}$}

Em função da natureza da espécie propagante pode-se estabelecer uma classificação dos tipos de polimerização em cadeia: polimerização radicalar ${ }^{[10]}$, polimerização catiônica ${ }^{[10]}$, polimerização aniônica ${ }^{[21]}$, e polimerização por coordenação ${ }^{[20]}$. Também existem alguns relatos na literatura sobre a polimerização aniônica e por coordenação ${ }^{[22]}$.

Em termos gerais, polimerizações por crescimento em cadeia caracterizam-se por serem processos rápidos nos quais se obtém polímeros de alta massa molar muito rapidamente, chegando a conversões próximas de $100 \%$. No decorrer da polimerização há sempre uma mistura de polímero e monômero, ao contrário das polimerizações por crescimento em etapas, nas quais o aumento da massa molar ocorre durante todo o tempo de evolução da reação. Na polimerização em cadeia existem, geralmente, quatro processos cinéticos: Iniciação, no qual se criam as espécies ativas às quais se unirão posteriormente outras unidades do monômero no processo de Propagação, o processo de Terminação no qual ocorre interação entre espécies ativas combinando-se ou desproporcionandose, e a transferência de cadeia. Em alguns sistemas de polimerização observa-se que as massas molares são menores que as esperadas teoricamente levando-se em conta apenas as reações de terminação por combinação e desproporção. Isto se deve a reações de transferência de cadeia na qual a atividade da cadeia em crescimento passa a outra espécie presente no meio, terminando a cadeia prematuramente e formando outra 
espécie capaz de iniciar a polimerização. O efeito da transferência de cadeia sobre a velocidade de polimerização dependerá da magnitude da velocidade de re-iniciação quando comparada àquela da espécie iniciadora original.

As reações de polimerização em cadeia podem ocorrer em meio homogêneo, isto é, uma só fase (polimerização em solução ou em massa); ou em meio heterogêneo, duas ou mais fases (polimerização em suspensão ou em emulsão).

Nas reações de fotopolimerização por cadeias, a etapa de iniciação é uma reação fotoquímica que conduz à formação de uma espécie reativa. O sistema fotoiniciador mais simples é aquele no qual algum composto do sistema sofre excitação eletrônica por absorção de luz e em seguida decompõe-se em espécies ativas. Este é o caso dos chamados fotoiniciadores por fragmentação. Em outros sistemas o composto excitado eletronicamente (fotoiniciador) interage com um segundo composto (co-iniciador) por transferência de energia ou reações redox para formar as espécies ativas. $\mathrm{O}$ fotoiniciador também pode ser excitado por transferência de energia de outra molécula, denominada fotossensibilizador, que é a que absorve luz e ativa o sistema iniciador, permanecendo inalterada até o final do processo ${ }^{[23]}$.

\section{Cinética de Fotopolimerização Radicalar [20,24]}

As equações a seguir descrevem os processos cinéticos da polimerização em cadeia via radical livre, considerando a produção de radicais livres tanto por fragmentação como por reações bimoleculares.

Iniciação: produção de radicais livres R' após absorção de luz, seguida de adição a uma primeira molécula de monômero M para produzir as espécies iniciadoras da cadeia, M* (radical iniciador ou radical primário)

$$
\begin{array}{lc}
\mathrm{C}+h v \stackrel{k_{d}}{\longrightarrow} \rightarrow \mathrm{R}^{\bullet} & \mathrm{R}_{\mathrm{d}}=k_{d}[\mathrm{C}] \\
\mathrm{R}^{\bullet}+\mathrm{M} \stackrel{k_{i}}{\longrightarrow} \mathrm{M}^{\bullet} & R_{i}=k_{i}[\mathrm{M}]\left[\mathrm{R}^{\bullet}\right]
\end{array}
$$

Propagação: crescimento de M* por adições sucessivas de moléculas do monômero $\mathrm{M}$, que é consumido rapidamente.

$$
\mathrm{M}_{n}^{\bullet}+\mathrm{M} \stackrel{k_{p}}{\longrightarrow} \mathrm{M}_{n+1}^{\bullet} \quad R_{p}=k_{p}\left[\mathrm{M}^{\bullet}\right][\mathrm{M}]
$$

Terminação: reação bimolecular entre dois macrorradicais

$$
\begin{aligned}
& \mathrm{M}_{n}^{\bullet}+\mathrm{M}_{m}^{\bullet} \stackrel{k_{t c}}{\longrightarrow} \mathrm{M}_{n+m} \quad R_{t}=k_{t}\left[\mathrm{M}^{\bullet}\right]^{2} \\
& \mathrm{M}_{n}^{\bullet}+\mathrm{M}_{m}^{\bullet} \stackrel{k_{t d}}{\longrightarrow} \mathrm{M}_{n}+\mathrm{M}_{m}
\end{aligned}
$$

A velocidades de polimerização, considerando algumas suposições e hipóteses do estado estacionário, é dada por

$$
R_{p}=k_{p}[\mathrm{M}]\left(\frac{R_{i}}{k_{t}}\right)^{1 / 2}
$$

\section{Cinética da Fotopolimerização Catiônica ${ }^{[20,25]}$}

A polimerização catiônica está essencialmente limitada a monômeros com substituintes contendo elétrons livres. Ela depende da formação e propagação de espécies iônicas positivas. A formação destes íons, com tempos de vida suficientemente longos para que haja propagação e que geralmente são estabilizados pelo solvente, fornecem produtos com altas massas molares. As polimerizações iônicas, em geral, são caracterizadas por uma variedade de modos de iniciação e terminação. Diferente da polimerização via radical, a terminação na polimerização iônica nunca envolve uma reação bimolecular entre duas cadeias poliméricas em propagação. A terminação ocorre por reação com o contra-íon, com o solvente, ou com outra espécie presente no meio reacional.

A adição de um próton ou um carbeno a átomos de oxigênio, nitrogênio ou grupos sulfônio ou fosfônio pode gerar espécies mais estáveis que os carbocátions durante o evento da polimerização devido ao caráter altamente nucleofílico do ligante ${ }^{[31]}$. Porém, as equações das etapas cinéticas da polimerização catiônica têm sido descritas para a dupla ligação carbono-carbono, como a seguir:

Iniciação: produção da espécie catiônica $\mathrm{C}^{+}$que se adiciona a uma primeira molécula de monômero $\mathrm{M}$ para produzir as espécies iniciadoras da cadeia, $\mathrm{CM}^{+}$

$$
\begin{aligned}
& \mathrm{I} \stackrel{h \tilde{\mathrm{o}}}{\longrightarrow} \mathrm{C}^{+} \mathrm{N}^{-} \\
& \mathrm{C}^{+} \mathrm{N}^{-}+\mathrm{M} \stackrel{k_{i}}{\longrightarrow} \mathrm{CM}^{+} \mathrm{N}^{-} \quad R_{i}=k_{i}[\mathrm{M}]\left[\mathrm{C}^{+} \mathrm{N}^{-}\right]
\end{aligned}
$$

onde $\mathrm{N}^{-}$representa o contra-íon.

Propagação: o par iônico (consistindo de um carbocátion e seu contra-íon negativo) produzido na etapa de iniciação se propaga por adições sucessivas de moléculas de monômero. Estas adições ocorrem por inserção de monômeros entre o carbocátion e seu contra-íon negativo.

$\mathrm{CM}_{\mathrm{x}}^{+} \mathrm{N}^{-}+\mathrm{M} \stackrel{k_{p}}{\longrightarrow} \mathrm{CM}_{\mathrm{x}} \mathrm{M}^{+} \mathrm{N}^{-} \quad R_{p}=k_{p}\left[\mathrm{CM}_{\mathrm{x}}^{+} \mathrm{N}^{-}\right][\mathrm{M}]$

Transferência de cadeia e Terminação: Várias reações levam à terminação da cadeia em crescimento, mas muitas destas reações não levam à terminação da cadeia cinética, já que uma nova espécie propagadora pode ser gerada no processo. Dentre estas reações as mais comuns são

a) Transferência de cadeia ao monômero. Dificilmente este tipo de reação deixa de ocorrer nas polimerizações. Ela envolve a transferência de um próton- $\beta$ do carbocátion ao monômero com formação de uma molécula de polímero e uma insaturação terminal.

$$
\mathrm{CM}_{\mathrm{X}} \mathrm{M}^{+} \mathrm{N}^{-}+\mathrm{M} \stackrel{k_{t r, \mathrm{M}}}{\longrightarrow} \mathrm{CM}^{+} \mathrm{N}^{-}+\mathrm{M}_{\mathrm{x}+1}
$$

b) Terminação espontânea ou transferência de cadeia ao contra-ion. Envolve a formação de um complexo iniciador - contraíon e uma molécula de polímero com insaturação terminal. 


$$
\mathrm{CM}_{\mathrm{X}} \mathrm{M}^{+} \mathrm{N}^{-} \stackrel{k_{t r}}{\longrightarrow} \mathrm{C}^{+} \mathrm{N}^{-}+\mathrm{M}_{\mathrm{x}+1}
$$

\section{c) Combinação com o contra-ion}

$$
\mathrm{CM}_{\mathrm{X}} \mathrm{M}^{+} \mathrm{N}^{-} \stackrel{k_{t}}{\longrightarrow} \mathrm{CM}_{\mathrm{x}} \mathrm{MN} \quad R_{t}=k_{t}\left[\mathrm{CM}_{\mathrm{x}} \mathrm{M}^{+} \mathrm{N}^{-}\right]
$$

Conforme indicado na equação 5 , a velocidades de polimerização é dada por

$$
R_{p}=\left(\frac{k_{p}}{k_{t}}\right) R_{i} \cdot[\mathrm{M}]
$$

\section{A influência da luz}

Tanto na polimerização radicalar como na iônica, a velocidade de iniciação em um sistema perfeitamente agitado é dada por

$$
R_{i=} \phi_{i}
$$

onde $\Phi_{i}$ é o rendimento quântico de iniciação, $I_{a}$ é a intensidade de luz absorvida (em unidades de fótons), que pode ser calculada a partir da Lei de Lambert-Beer

$$
I_{a}=I_{o}\left(1-10^{-\varepsilon[\mathrm{C}] l}\right)
$$

onde $\varepsilon$ é o coeficiente de extinção molar do cromóforo no comprimento de onda de irradiação, [C] é a concentração da espécie absorvente, $l$ é o caminho óptico; e $I_{o}$, a intensidade de luz incidente.

A eficiência de um sistema pode ser descrita pela velocidade de polimerização, $R_{p}$, ou pela velocidade de iniciação, $R_{i}$, as quais dependem das condições experimentais. De forma absoluta, para sua descrição podem ser utilizados os parâmetros a seguir

Rendimento quântico de iniciação $\left(\Phi_{i}\right)$, definido como o número de cadeias do polímero iniciadas por fóton absorvido:

$$
\Phi_{i}=\frac{R_{i}}{I_{a}}
$$

Rendimento quântico de polimerização $\left(\Phi_{m}\right)$, definido como o número de unidades do monômero polimerizadas, por fóton absorvido

$$
\Phi_{m}=\frac{R_{p}}{I_{a}}
$$

O rendimento quântico de iniciação leva em conta todos os processos que levam à formação das espécies procedentes da adição ao monômero, portanto depende do rendimento de cruzamento entre sistemas $\left(\Phi_{\text {isc }}\right)$, da eficiência dos radicais fotogerados e da velocidade dos diferentes processos envolvidos.

\section{Sistemas de fotopolimerização envolvendo corantes ${ }^{[26]}$}

Como, em geral, os componentes dos sistemas polimerizáveis não absorvem luz no comprimento de onda de interesse (visível e uv próximo) é necessário adicionar um componente, denominado fotoiniciador ou simplesmente iniciador, que absorva luz e conduza à formação de espécies reativas a partir de uma reação de seus estados excitados.

As reações mais importantes das moléculas de corantes envolvem processos de transferência de elétrons, produzindo espécies de vida curta, neutras, radicalares ou carregadas. Existem duas formas de gerar as espécies reativas iniciadoras: fotorredução do corante no estado excitado $\left(\mathrm{C}^{*}\right)$ por doadores de elétrons (:D) (equação 19), ou fotooxidação por aceptores de elétrons (A) (equação 20):

$$
\begin{aligned}
& \mathrm{C}^{*}+\mathrm{D} \stackrel{k_{q}}{\longrightarrow} \mathrm{C}^{-\bullet}+\mathrm{D}^{+\bullet} \\
& \mathrm{C}^{*}+\mathrm{H}-\mathrm{D} \stackrel{k_{q}}{\longrightarrow} \mathrm{H}-\mathrm{C}^{\bullet}+\mathrm{D}^{\bullet} \\
& \mathrm{C}^{*}+\mathrm{A} \stackrel{k_{q}}{\longrightarrow} \mathrm{C}^{+\bullet}+\mathrm{A}^{-\bullet}
\end{aligned}
$$

A formação de um determinado produto vai depender do potencial de oxidação do doador, do potencial de redução de aceptor, e da energia de excitação do correspondente estado excitado do corante.

\section{Métodos experimentais para a determinação da velocidade de polimerização}

As velocidades de fotopolimerização geralmente são estudadas usando as mesmas metodologias que aquelas aplicadas à polimerizações térmicas porém, monitorando-se adequadamente a influência da irradiação. As técnicas normalmente usadas para avaliar as polimerizações por via radical livre são geralmente aplicáveis às polimerizações iônicas. Alguns dos métodos têm a vantagem inerente de não necessitar parar a reação para determinar a porcentagem de conversão ${ }^{[2,20]}$.

\section{Gravimetria}

Um dos métodos mais simples é a medida gravimétrica ${ }^{[27]}$. Após determinado tempo de irradiação do sistema reacional o polímero é precipitado em um não-solvente e a porcentagem de conversão calculada após secagem. Esta metodologia tem sido aplicada a sistemas de fotopolimerização via radicalar homogêneos ${ }^{[28]}$ e heterogêneos ${ }^{[29]}$, e os resultados sempre correspondem a integrações da velocidade sobre um determinado tempo. Os valores obtidos podem ser utilizados, sobretudo, em avaliações relativas. A Figura 1 ilustra o método para a fotopolimerização via radical livre do metacrilato de metila em solução micelar aquosa de dodecilsulfato de sódio, utilizando o corante tionina como iniciador e trietilamina como co-iniciador ${ }^{[29]}$.

A Figura 2 mostra a variação da porcentagem de conversão com a concentração da amina, após 5 minutos de irradiação. 


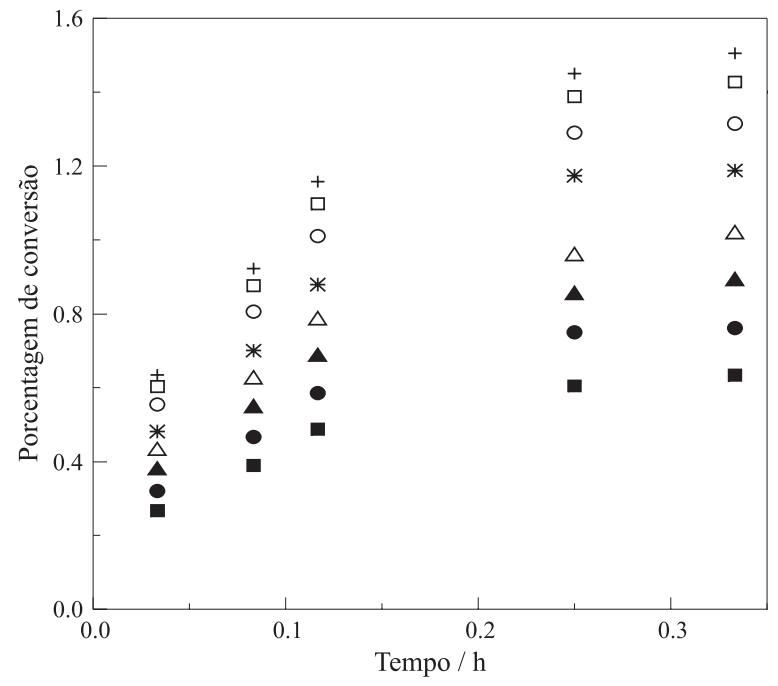

Figura 1. Porcentagem de conversão versus o tempo de irradiação para a polimerização de metacrilato de metila em dodecilsulfato de sódio obtida em presença do sistema tionina / trietilamina nas concentrações de

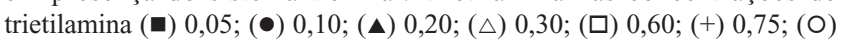
0,$90 ;(*) 1,0$ mol.L ${ }^{-1}$

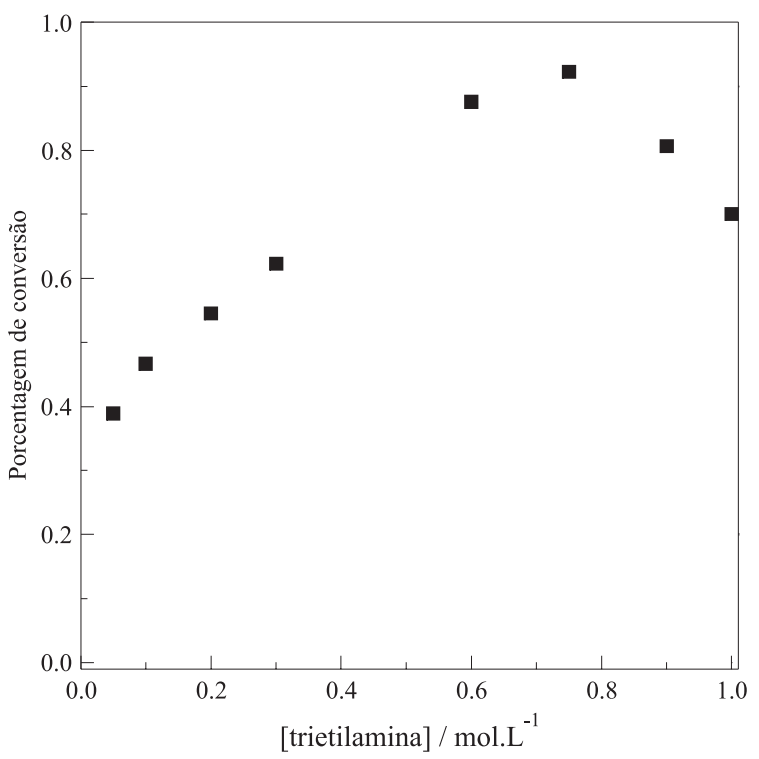

Figura 2. Porcentagem de conversão versus concentração de trietilamina, para a fotopolimerização de metacrilato de metila em dodecilsulfato de sódio pelo sistema tionina / trietilamina, após 5 minutos de irradiação.

Gráficos deste tipo fornecem informações diferentes daquelas da Figura 1 para os mesmos dados obtidos por gravimetria e podem ser indicativos se existem processos que diminuem a formação dos radicais iniciadores da polimerização ${ }^{[29,30]}$.

$\mathrm{O}$ avanço da polimerização pode ser acompanhado experimentalmente, de maneira indireta, pela variação de alguma propriedade do monômero que difira daquela do polímero como, por exemplo, solubilidade, densidade, índice de refração, espectro de absorção ${ }^{[20]}$. A variação da viscosidade e da micropolaridade do meio, enquanto avança a reação, podem ser determinadas usando sondas fluorescentes, as quais mudam suas propriedades espectroscópicas dependendo do meio onde estão localizadas ${ }^{[31]}$. Estas medidas podem ser relacionadas a velocidade de polimerização pela utilização de relações apropriadas.

O conhecimento da cinética de reação é de importância fundamental e um dos problemas enfrentados é a utilização de uma metodologia que permita estudar a cinética da fotopolimerização em tempo real, que pode ocorrer em tempos da ordem dos milissegundos. Neste sentido têm sido utilizadas técnicas como, por exemplo, dilatometria ${ }^{[30,32-35]}$, espectroscopia fotoacústica ${ }^{[36]}$, espectroscopia de infravermelho

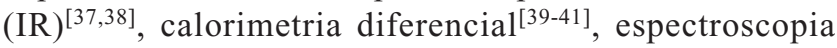
$\operatorname{Raman}^{[42,43]}$, espalhamento de luz ${ }^{[44]}$, e ressonância paramagnética eletrônica ${ }^{[45,46]}$.

\section{Fotodilatometria}

A dilatometria utiliza as variações de volume que acontecem durante a polimerização devido a que a densidade dos polímeros é normalmente maior que a dos monômeros a partir dos quais são formados. Assim, a velocidade de polimerização $\left(R_{p}\right)$ pode ser seguida observando-se a contração em volume da massa reacional durante a polimerização. Paralelamente à medida de contração de volume da solução, é determinada a intensidade de luz absorvida pela amostra mediante a leitura radiométrica, calibrada previamente com uma solução do actinômetro no interior do dilatômetro. A técnica permite obterse valores confiáveis já que o sistema é constantemente agitado, há controle contínuo da temperatura e da intensidade da luz incidente. O método utiliza um equipamento semelhante ao da Figura 3, montado de forma a permitir a irradiação do dilatômetro simultaneamente à tomada de medidas de contração de volume no capilar com um catetômetro ${ }^{[33]}$. O sistema em questão é composto das seguintes partes

a) lâmpada de $\mathrm{Hg}(\mathrm{Xe})$ de $200 \mathrm{~W}$ alojada em um compartimento contendo um sistema óptico de focalização, com sua correspondente fonte de arranque e alimentação;

b) filtro de infravermelho termostatizado;

c) lente focalizadora;

d) monocromador de alta resolução com sistema de focalização e controle das fendas de entrada e saída;

e) camisa de termostatização com sistema de agitação magnética e termômetro;

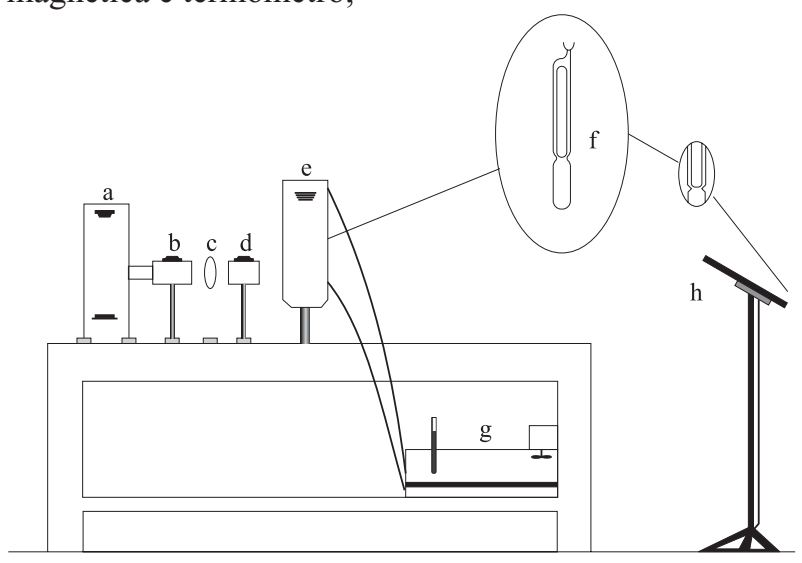

Figura 3. Esquema do sistema de fotodilatometria. 
f) dilatômetro de vidro (dentro da camisa de termostatização);

g) banho termostático / circulador;

h) catetômetro de precisão.

Após selecionar o comprimento de onda desejado, as irradiações são realizadas sob agitação com um imã no interior do dilatômetro. $\mathrm{O}$ dilatômetro é alojado no interior de uma camisa de termostatização, ficando completamente submergido e assegurando um perfeito controle de temperatura durante todo o experimento. $\mathrm{O}$ controle rigoroso da temperatura é fundamental já que os parâmetros cinéticos se obtêm através da contração de volume que se produz no processo de polimerização e em geral, as polimerizações são exotérmicas. $\mathrm{O}$ dilatômetro deve ser calibrado previamente com tetracloreto de carbono para conhecer exatamente a área do capilar.

Devido à importância do conhecimento da intensidade de luz incidente nos experimentos fotoquímicos, é necessário determinar a actinometria do sistema. Como ilustração, se exemplifica a utilização do actinômetro ferrioxalato de potássio, $\mathrm{K}_{2}\left[\mathrm{Fe}\left(\mathrm{C}_{2} \mathrm{O}_{4}\right)_{3}\right]$ em presença de fenantrolina, o qual é sensível na faixa de comprimento de onda $(\lambda)$ de $254-500 \mathrm{~nm}^{[47]}$. $\mathrm{O}$ rendimento quântico $(\phi)$ do actinômetro irradiado e a intensidade de luz incidente $\left(\mathrm{I}_{\mathrm{o}}\right)$ devem ser determinados.

Quando a solução de ferrioxalato é fotolisada, os íons $\mathrm{Fe}^{3+}$ são reduzidos a $\mathrm{Fe}^{2+}$ segundo

$$
\mathrm{Fe}^{3+} \stackrel{h i ́}{\longrightarrow} \mathrm{Fe}^{2+}+\text { produtos }
$$

Embora ambos íons formem complexos com a fenantrolina, somente o complexo formado com os íons $\mathrm{Fe}^{2+}$ absorve a $510 \mathrm{~nm}$. A intensidade da luz incidente pode ser obtida da equação

$$
I_{o}=\frac{n\left(\mathrm{Fe}^{2+}\right)}{\phi . t .\left(1-10^{-\varepsilon c l}\right)}
$$

sendo $n\left(\mathrm{Fe}^{2+}\right)$, o número de íons formados durante a fotólise, que pode ser calculado

$$
n\left(\mathrm{Fe}^{2+}\right)=\frac{v_{1} \cdot v_{3} \cdot \Delta \mathrm{A}}{v_{2} \cdot l \cdot \varepsilon}
$$

onde

$I_{o} \rightarrow$ intensidade da luz incidente (Einstein/s)

$\phi \rightarrow$ rendimento quântico do actinômetro irradiado

$t \rightarrow$ tempo de irradiação (s)

$\varepsilon \rightarrow$ absortividade molar do complexo de $\mathrm{Fe}^{2+}\left(1,11 \times 10^{4} \mathrm{~L} /\right.$ mol.cm) $)^{(47)}$

$\Delta \mathrm{A} \rightarrow$ variação da absorbância da solução a $510 \mathrm{~nm}$

$l \rightarrow$ caminho óptico da cela de irradiação $(\mathrm{cm})$

$v_{l} \rightarrow$ volume da solução de actinômetro irradiado $(\mathrm{mL})$

$v_{2} \rightarrow$ volume da alíquota tomada para análise $(\mathrm{mL})$

$v_{3} \rightarrow$ volume final após diluição de $v_{2}(\mathrm{~mL})$

$c \rightarrow$ concentração do actinômetro ( $\mathrm{mol} / \mathrm{L}$ )

$\left(1-10^{-\varepsilon c l}\right) \rightarrow$ fração de luz incidente absorvida pela amostra
A velocidade de polimerização $\left(R_{p}\right)$ é calculada segundo a equação

$$
R_{p}=\frac{h}{\mathrm{~F} \times t \times f}[\mathrm{M}]
$$

onde $h$ é a variação de altura no capilar medida com o catetômetro no tempo $t$; $f$ é a fração de volume do monômero na solução; [M], a concentração de monômero e F, a contração de volume relacionada com as densidades do monômero e do polímero $\left[\mathrm{F}=\left(\mathrm{d}_{\mathrm{p}}-\mathrm{d}_{\mathrm{m}}\right) / \mathrm{d}_{\mathrm{p}}\right]$. As velocidades de polimerização são correlacionadas com as variações na intensidade de luz incidente e as porcentagens de conversão calculadas da relação entre o produto da velocidade de polimerização pelo tempo de irradiação dividido pela concentração do monômero.

As velocidades de fotopolimerização $\left(R_{p}\right)$ do monômero tetraidrofurano (THF), utilizando concentrações constantes do sensibilizador tioxantona e variando a concentração do iniciador hexafluoroarsenato de trifenilsulfônio são determinadas

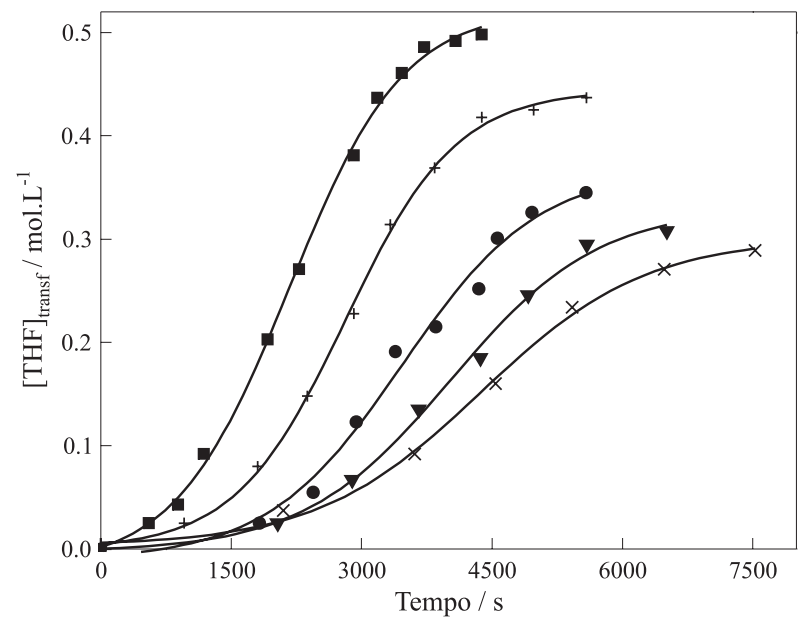

Figura 4. Concentração de THF transformado em função do tempo para a fotopolimerização em presença do sistema tioxantona / hexafluoroarsenato

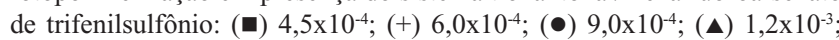
(x) $1,6 \times 10^{-3} \mathrm{M}$.

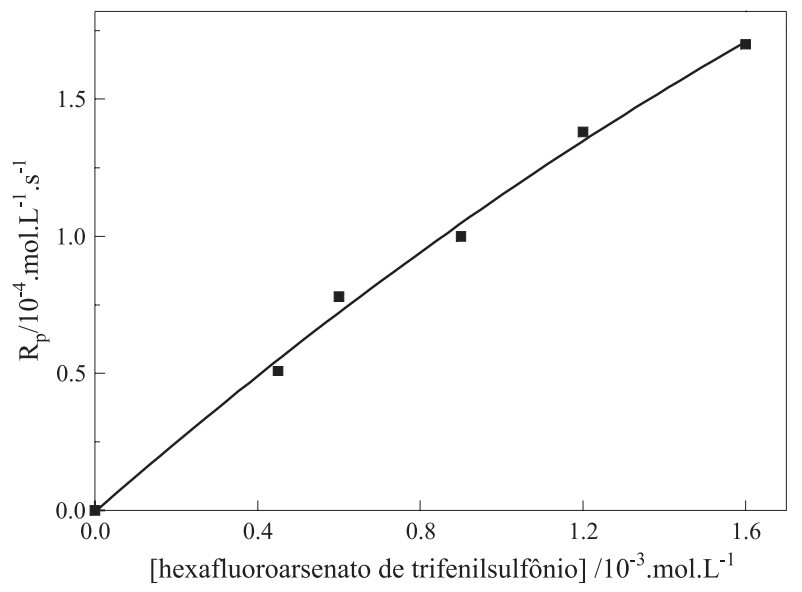

Figura 5. Velocidade de polimerização $\left(R_{p}\right)$ do THF para diferentes concentrações de hexafluoroarsenato de trifenilsulfônio. 


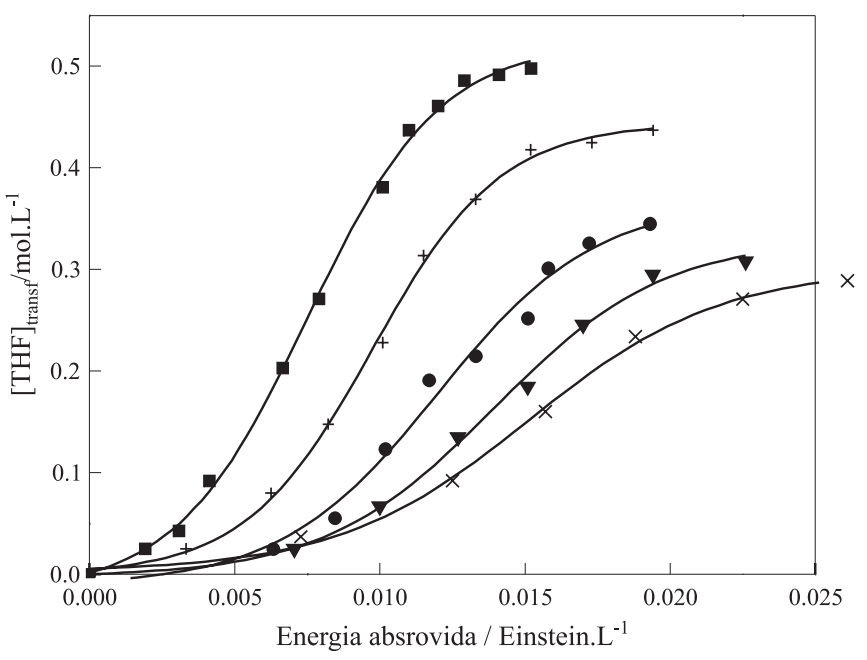

Figura 6. Concentração de THF transformado em função da energia absorvida (sistema fotoiniciador tioxantona / hexafluoroarsenato de

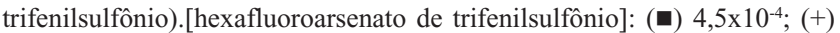
$\left.6,0 \times 10^{-4} ; \bullet \bullet\right) 9,0 \times 10^{-4} ;(\nabla) 1,2 \times 10^{-3} ;(x) 1,6 \times 10^{-3} \mathrm{M}$.

da parte linear das inclinações de gráficos de concentração do THF transformado versus tempo como mostrado na Figura $4^{[33]}$. Com estes dados é montada a Figura 5 que mostra o comportamento da velocidade de polimerização $\left(R_{p}\right)$ com a concentração do iniciador (hexafluoroarsenato de trifenilsulfônio). Por outro lado, o rendimento quântico de polimerização $\left(\Phi_{m}\right)$ pode ser calculado da parte linear de gráficos de concentração do monômero transformado versus energia absorvida (Figura 6).

Estes resultados demonstram a variedade de determinações possíveis através de técnica dilatométrica em comparação à gravimétrica.

\section{Espectroscopia de infravermelho em tempo real (RTIR)}

A espectroscopia de infravermelho em tempo real (RTIR) ou espectroscopia de infravermelho por transformada de Fourier (FTIR) com reflexão especular permite que a velocidade de desaparecimento da dupla ligação do monômero, no caso de monômeros vinílicos, seja monitorada em tempo real podendo assim avaliar-se quantitativamente a evolução do processo de polimerização. Neste método as amostras são espalhadas sobre um filme de poliéster recoberto de alumínio e irradiadas através de fibras ópticas durante um tempo pré-determinado usando uma lâmpada apropriada. A velocidade de fotocura é determinada pelo monitoramento do desaparecimento da banda de deformação fora do plano da dupla ligação vinílica a $\sim 815 \mathrm{~cm}^{-1}$. A intensidade desta banda deve ser comparada com outra não afetada pela polimerização para compensar as variações na espessura do filme fotocurável devido à reação e posição da amostra, como por exemplo, uma banda correspondente ao estiramento da carbonila de éster a $\sim 1735 \mathrm{~cm}^{-1}$.

A evolução do espectro de infravermeho durante a fotocura da resina Actilane 420 (bisfenol-A-dimetacrilato etoxilatado) em presença do sistema fotoiniciador tionina / amina está mostrado na Figura 7. Neste caso foi utlizado um acessório de reflexão especular adaptado de forma de permitir a irradiação simultaneamente ao registro dos espectros.

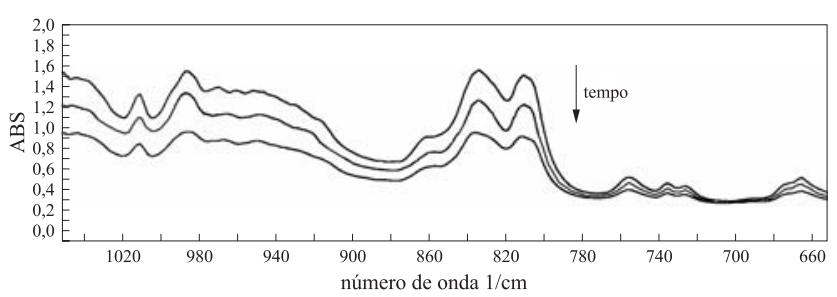

Figura 7. Espectros de FTIR para a conversão do pré-polímero comercial Actilane $420 \mathrm{em}$ presença da tionina / trietanolamina $0,35 \mathrm{M}$, utilizando uma lâmpada de mercúrio de alta pressão de $100 \mathrm{~W}$.

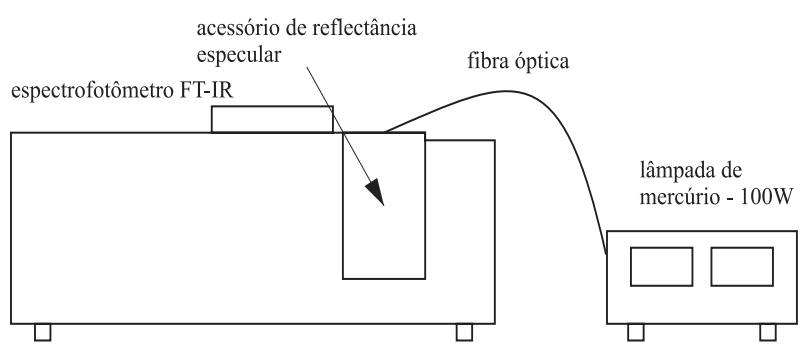

Figura 8. Diagrama mostrando o layout dos componentes do sistema de FTIR

A montagem de um equipamento para este fim está esquematizada na Figura 8. Também é bastante comum o uso de acessórios de refletância difusa nos equipamentos de FTIR.

O grau de cura está dado por

$$
\% \text { cura }=\left\{\left[1-\left(A_{815} / A_{1735}\right)_{t}\right] /\left(A_{815} / A_{1735}\right)_{0}\right\} \times 100
$$

onde $A$ é a absorbância, e os índices 0 e $t$ se referem aos tempos de irradiação inicial e a tempo $t$, respectivamente. $\mathrm{Na}$ Figura 9 está mostrada a conversão da Actilane versus a concentração de amina após 7 minutos de irradiação, exemplificado para o caso da trietanolamina.

Reações de polimerização in situ podem ser acompanhadas por espectroscopia Raman desde que haja uma variação de intensidade da banda característica do monômero ou do

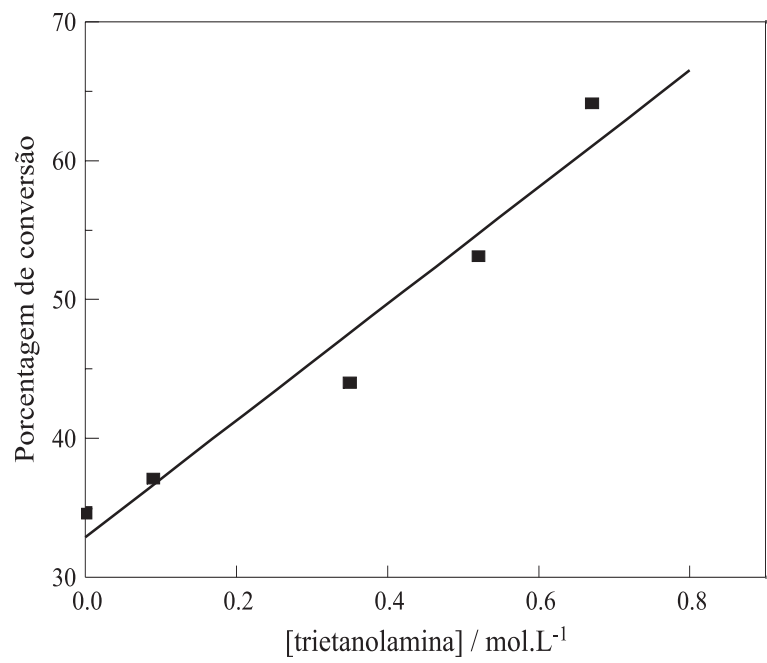

Figura 9. Conversão do pré-polímero comercial Actilane 420 em função da concentração de trietanolamina, utilizando o iniciador tionina, após 7 minutos de irradiação com uma lâmpada de $\mathrm{Hg}$ de alta pressão de 100W. 
polímero durante o progresso da polimerização. Os deslocamentos Raman estão associados a determinados modos normais de vibração da mesma maneira que estão as absorções no infravermelho. A técnica possui a vantagem de que os espectros podem ser obtidos através das paredes de vidro do compartimento reacional, usando fibras ópticas para a deteç̧ão ${ }^{[42]}$.

A espectroscopia Raman pode ser usada, por exemplo, para estudar a cinética de polimerização de monômeros vinílicos, nos quais o grupo $\mathrm{C}=\mathrm{C}$ exibe um comportamento que pode ser monitorado, como o metacrilato de metila ${ }^{[48]} \mathrm{e}$ o estireno ${ }^{[49]}$, monômeros passíveis de fotopolimerização em presença de sistemas iniciador/co-iniciador ${ }^{[28-30,50]}$.

\section{Calorimetria}

Praticamente todos os processos de polimerização são exotérmicos, de forma que o calor desprendido pode ser relacionado com a conversão do monômero em polímero. Basicamente, a calorimetria diferencial, DSC, mede a velocidade de aquecimento observada, assim como o calor total produzido na reação química. Esta técnica apresenta a vantagem de fornecer grande quantidade de informação utilizando pequenas quantidades de amostra. $\mathrm{O}$ método calorimétrico para o estudo da polimerização fotoiniciada foi introduzido há alguns anos ${ }^{[51]}$ e contribuiu para um avanço importante na técnica de fotocura de recobrimentos tradicionalmente difíceis de estudar em profundidade.

A curva exotérmica obtida no calorímetro corresponde ao desprendimento de calor $(\mathrm{d} H / \mathrm{d} t)$ versus o tempo de irradiação. A área da curva exotérmica, $A$, corresponde ao calor total de polimerização da amostra

$$
\Delta H_{\text {polim }}=A
$$

Levando-se em conta que a quantidade de calor desprendido é proporcional ao número de moles de monômero polimerizado, $n$, sendo $H$ o calor desprendido ao tempo $t, n_{o}$ o número total de moles iniciais de monômero, e derivando com respeito ao tempo, se obtêm

$$
-\frac{\mathrm{d} n}{\mathrm{~d} t}=-\frac{n_{O}}{A} \frac{d H}{\mathrm{~d} t}
$$

e a equação da velocidade de polimerização, semelhante às equações 5 e 11 para as polimerizações aqui descritas, pode ser escrita como

$$
-\frac{\mathrm{d} n}{\mathrm{~d} t}=k n
$$

Considerando o número médio de moles sem reagir, $n^{\prime}$, a tempo $t n^{\prime}=n_{O}-\frac{n_{O} H}{A}$ e particularizando o valor de $n$ a tempo $t, n$ ', obtém-se o valor de $k$

$$
k=\frac{-(\mathrm{d} H / \mathrm{d} t)}{A-H}
$$

onde $\mathrm{d} H / \mathrm{d} t(\mathrm{em} \mathrm{mcal} / \mathrm{s})$ é a ordenada da curva na fração de conversão $H / A$ escolhida e $[A-H]$ (mcal) o calor residual de polimerização do monômero sem reagir. Assim, a partir da integração da curva a conversão total, ou em cada caso a conversão teto ${ }^{[8]}$, se determina a área total, $A$, e calculando as áreas parciais, $H$, nas unidades adequadas (levando em conta o calor de polimerização da unidade monomérica e o peso da amostra) pode-se fazer representações da concentração de monômero transformado frente ao tempo, cuja inclinação fornecerá a velocidade de polimerização $R_{p}$.

As determinações calorimétricas são feitas usando sistemas como o mostrado na Figura 10, que consiste em um calorímetro comercial modificado, ${ }^{[52,53]}$ incorporando um acessório que permite a irradiação da amostra fotopolimerizável simultaneamente à obtenção das curvas exotérmicas.

A. fonte de luz. lâmpada de alta pressão de mercúrio $(100 \mathrm{~W})$, provista de lentes focalizadoras de quartzo e um espelho de alumínio;

B. cilindro de alumínio onde estão colocados vários filtros (neutro, de infravermelho, e de interferência). O último para escolher o comprimento de onda requerido para a reação;

C. conector de fibras ópticas;

D. duplo feixe de fibras ópticas flexíveis de quartzo;

E. cabeçal do calorímetro;

F. cavidade do calorímetro conectada a uma unidade de aquisição e tratamento de dados.

A intensidade incidente é determinada medindo o aumento de temperatura devido à absorção de luz no poço de uma cápsula idêntica às utilizadas para irradiação das amostras $(10 \mu \mathrm{L}$ de volume de amostra), recoberta com uma fina camada de grafite. Deve-se dispor de uma cápsula de alumínio correspondente com o objetivo de obter por referência o valor da energia. A Figura 11 representa a seção transversal da cápsula de alumínio.

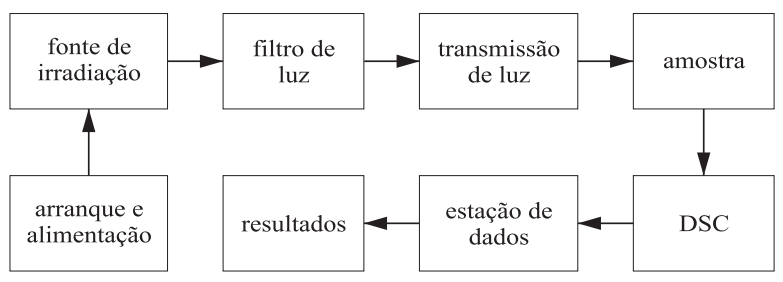

Figura 10. Esquema do fotocalorímetro diferencial comercial, modificado para ser usado em cinéticas de fotopolimerização.

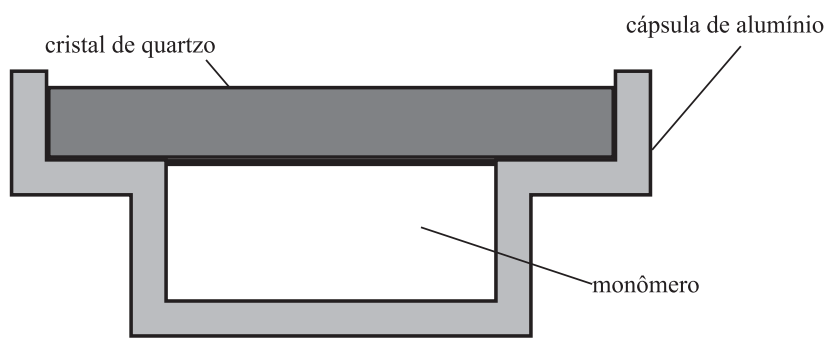

Figura 11. Seção transversal da cápsula de alumínio. 


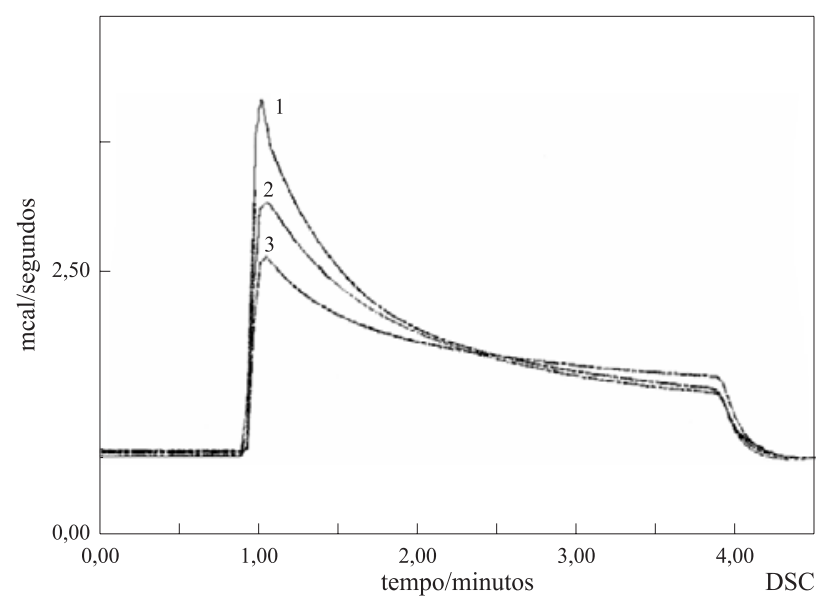

Figura 12. Exoterma da polimerização da acrilamida fotoiniciada pelo sistema tionina / trietanolamina em função do tempo. [trietanolamina]: (1) 0,$3 ;$ (2) 0,$2 ;(3) 0,1 \mathrm{M}$.

A Figura 12 mostra os valores da integração para as curvas obtidas do começo da irradiação até o corte da luz para a fotopolimerização do monômero acrilamida em solução aquosa usando o sistema fotoiniciador tionina / trietanolamina, com irradiações isotérmicas a $40{ }^{\circ} \mathrm{C}$.

Sob as condições experimentais das medidas de fotocalorimetria (meio aeróbico) as velocidades de polimerização são influenciadas por vários fatores, tais como o rápido aumento da viscosidade do meio e as altas intensidades de irradiação no volume do porta-amostras do calorímetro. Por isso os dados devem ser considerados como relativos e não absolutos.

A eficiência da tionina como fotoiniciador do monômero acrilamida pode ser comprovada pelas velocidades de polimerização, $R_{p}$. O gráfico das concentrações do monômero acrilamida transformado versus o tempo de irradiação a várias concentrações de trietanolamina (Figura 13) permitem o cálculo das velocidades de polimerização, $R_{p}$, a partir da parte inicial dos gráficos, conforme mostrado na Figura 14.

Uma aplicação específica desta metodologia é o estudo cinético de sistemas de resinas epóxi utilizadas em compósitos

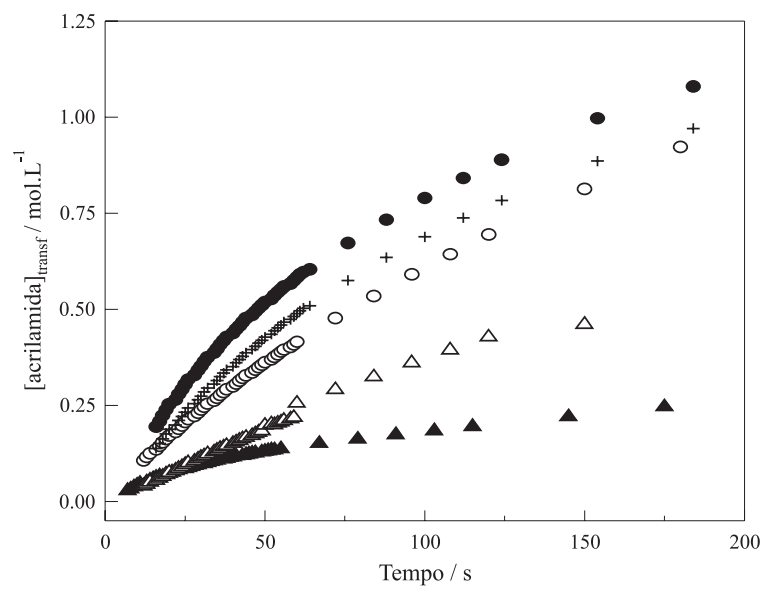

Figura 13. Concentração de acrilamida fotopolimerizada em presença do sistema tionina / trietanolamina a $40^{\circ} \mathrm{C}$ em solução aereada. $\lambda_{\text {irrad }}: 600 \mathrm{~nm}$. [TEA]: $(\Delta) 0,050 ;(\triangle) 0,10 ;(0) 0,15 ;(+) 0,20 ;(\bullet) 0,30 \mathrm{M}$.

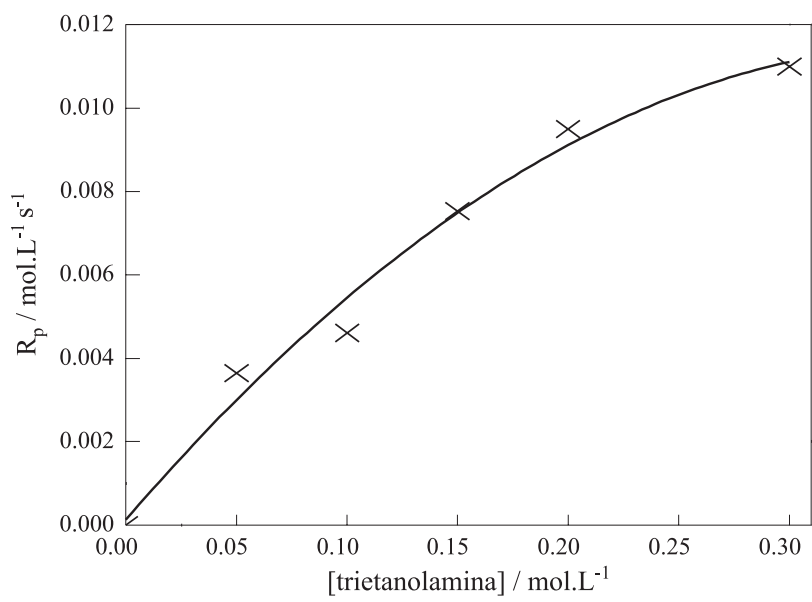

Figura 14. Velocidade de fotopolimerização da acrilamida em água a $40^{\circ} \mathrm{C}$, em presença de oxigênio, em função da concentração de trietanolamina, utilizando tionina como iniciador.

pré-impregnados (moldados em autoclave na conformação de elementos estruturais). A tecnologia de fabricação destes compostos depende do conhecimento da cinética de cura destas resinas por técnicas precisas como por via DSC ${ }^{[54]}$.

\section{Outros métodos}

A técnica de ressonância paramagnética eletrônica (EPR) permite a avaliação da quantidade de radicais livres presentes no processo de fotopolimerização, durante e após a irradiação. O método envolve a irradiação por uma fonte de luz apropriada, posicionada em uma janela do espectrofotômetro, focalizada sobre a amostra. Este método permite a obtenção de resultados cinéticos relativos à fotopolimerização, como exemplificado para o caso de resinas dentárias fotopolimerizáveis contendo carga de zircônia e sílica, irradiadas com luz azul, indicando que a polimerização continua ainda depois de cessar a irradiação ${ }^{[55]}$.

O efeito fotoacústico consiste basicamente na geração de ondas sonoras a partir da absorção de luz. Estas ondas sonoras podem ser originadas por diferentes mecanismos, o principal dos quais é a difusão térmica descrita a seguir. Inicialmente, um feixe de luz modulada atinge a superfície da amostra (mantida dentro de uma célula fechada contendo gás). A amostra absorve parte da luz e libera calor, que se propaga através da amostra causando o aquecimento do gás. O aquecimento periódico do gás produz ondas de pressão que são detectadas por um microfone acoplado à célula.

No processo de polimerização, as variações na taxa de calor produzido estão relacionadas com a conversão do monômero em polímero. A intensidade do sinal fotoacústico depende da quantidade de calor gerada na amostra, que por sua vez varia com a intensidade de luz, o coeficiente de absorção óptica e a eficiência de conversão da luz absorvida em calor pela amostra ${ }^{[56]}$. A copolimerização aleatória de poli(3-hidroxibutirato-co-3-hidroxivalerato) foi estudada desta forma. Além de informações sobre a velocidade de plastificação interna, foram obtidos dados sobre condutividade e difusividade térmica ${ }^{[57]}$. 
O número de cadeias poliméricas presentes como partículas coloidais é proporcional à quantidade de monômero polimerizado. Como estas partículas espalham a luz, medidas da intensidade do espalhamento permitem avaliar o grau de avanço de reações, mesmo a baixas taxas de conversão, já que existe uma correlação direta entre a massa precipitada do polímero e o grau de espalhamento ${ }^{[44]}$.

\section{Conclusões}

A utilização de métodos para determinar velocidades de fotopolimerização de forma geral é um assunto que vem sendo bastante estudado na literatura. Os principais pontos de interesse nesta área envolvem o aprimoramento de métodos para estudar em tempo real a cinética da polimerização, que ocorre em intervalos de milissegundos, ou, pelo menos, avaliações relativas que permitam determinações através de integrações da velocidade em um determinado tempo. Os métodos que têm sido utilizados geralmente são os mesmos usados em polimerizações térmicas porém, mantendo alguns cuidados específicos necessários para sistemas submetidos à irradiação UV-visível.

Este artigo foca sua discussão em sistemas investigados pelo Grupo de Fotoquímica - IQSC - USP, alguns ainda não publicados, e cita alguns sistemas investigados por outros pesquisadores.

\section{Agradecimentos}

Os autores agradecem à FAPESP (Fundação de Amparo à Pesquisa do Estado de São Paulo) pelo suporte financeiro.

\section{Referências Bibliográficas}

1 Allen, N. S. \& Rabek, J. F. - "New Trends in the Photochemistry of Polymers", Elsevier Applied Science Publishers, London, (1985).

2 Braun, A. M.; Maurette, M. T. \& Oliveros, E. "Photochemistry Technology", John Wiley, Inglaterra, (1991).

3 Catalina, F.; Martinez-Utrilla, R. \& Sastre - R., Polym. Bull., 8, p.369, (1982).

4 Hodge, P. \& Sherrington, D.C. - "Polymer Supported Reactions in Organic Synthesis", John Wiley, Chichester, (1980).

5 Randy, B. \& Rabek, J.F. - "Photodegradation, Photooxidation and Photostabilization of Polymers", John Wiley, London, (1975).

6 Catalina, F.; Allen, N.S. \& Chirinos-Padron, A. - Rev. Plást. Mod., 415, p.99, (1991).

7 Allen, N.S. - "Photopolymerization and Photoimaging Science and Technology", Elsevier Applied Science, England, (1989).
8 Pappas, P. - "UV Curing Science and Technology”, Technology Marketing Corp., Connecticut, USA, (1978).

9 Baratieri, L.B. - "Estética- Restaurações Adesivas Diretas em Dentes Anteriores Fraturados", Santos Livraria Editora - Quintessence Books -São Paulo, (1995).

10 Dietliker, R.K. - "Chemistry and Technology of UV and EB Formulation for Coating, Inks, and Paints", v.3, SITA Technology Ltd., London, (1991).

11 Fouassier, J.P. \& Lougnot, D.J. - "Radiation Curing of Polymer Materials", v.5, ACS Symp. Ser. 417, (1990).

12 Allen, N.S.; Edge, M.; Bellobono, I.R. \& Selli, E. - "Current Trends in the Photochemistry of Polymers", Ellis Horwood, London, (1995).

13 Böttcher, H.; Bendig, J.; Fox, M.A.; Hopf, G.H. \& Timpe, H.-J. - "Technical Applications of Photochemistry”, Dt. Verl. für Grundstoff., Leipzig, (1991).

14 Timpe, H.-J. \& Rajendran, A.G. - Eur. Polym. J., 27, p.77, (1991).

15 Athey, R.D. - "Emulsion Polymer Technology", Marcel Decker, New York, (1991).

16 Mergout, K.; Yabe, T.; Ishioka, S.; Kato, K. \& Esumi, K. Bull. Chem. Soc. Jpn., 59, p.3019, (1986).

17 Hourston, D.J.; Satgurunathan, R. \& Varna, H. - J. Appl. Polym. Sci., 31, p.1955, (1986).

18 Tredgold, R.H. - J. Chim. Phys., 85, p.1079, (1988).

19 Guyot, A. \& Tauer, K. - Adv. Polym. Sci., 111, p.45, (1994).

20 Odian, G. - "Principles of Polymerization", McGraw-Hill, New York, (1982).

21 Paul, R.B.; Kelly, J.M.; Pepper, D.C. \& Long, C. - Polymer, 38, p2011, (1997).

22 Fouassier, J.-P. - "Photoinitiation, Photopolymerization and Photocuring- Fundamentals and Applications", Hanser / Gardner Publications, Inc., Cincinnati, (1995).

23 Koizumi, M.; Kato, S.; Mataga, N.; Matsura,T. \& Usui, Y. - "Photosensitized Reactions", Kagakudojin Publishing Co., Inc., Kioto, Japon, (1978).

24 Rabek, J.F. - "Photochemistry and Photophysicy", v.4, CRC Press, Inc., USA, (1991).

25 Smid, J. \& Fish, D. - "Encyclopedia of Polymer, Science and Engineering", v.2, Wiley, New York, (1988). p.729.

26 Scaiano, J.C. - "Handbook of Organic Photochemistry", v.2, CRC Press Inc., Florida, (1989).

27 Lenka, S. \& Mohanty, J. - J. Polym. Photochem., 7, p.447, (1986).

28 Neumann, M.G. \& Rodrigues, M.R. - Polymer, 39, p.1657, (1998). 
29 Rodrigues, M.R.; Catalina, F. \& Neumann, M.G. - J. Photochem. Photobiol. A: Chem., 124, p.29, (1999).

30 Rodrigues, M.R.; Catalina, F. \& Neumann, M.G. - J. Photochem. Photobiol. A: Chem., 127, p.147, (1999).

31 Paczkowski, J.; Neckers, D. C. - J. Polym. Sci., Part A: Polym. Chem., 31, p.841; (1993).

32 Encinas, M.V.; Lissi, E.; Majmud, C. \& Cosa, J.C.Macromolecules, 26, p.6284, (1993).

33 Rodrigues, M.R. \& Neumann, M.G. - Macromol. Chem. Phys., 202, p.2776, (2001).

34 Rodrigues, M.R. \& Neumann, M.G. - J.Polym.Sci. A: Pol. Chem., 39, p.46, (2001).

35 Neumann, M.G. \& Rodrigues, M.R. - J. Braz. Chem. Soc., 14, p.76 (2003)

36 Small, R. D.; Ors, J. A. \& Royce, B. S. - ACS Symp.. Ser., 242, p.325, (1984).

37 Decker, C. \& Moussa K. - Macromolecules, 22,p.4455, (1989).

38 Decker C. - J. Polym. Sci., Polym. Chem. Ed., 21, p.2451, (1983).

39 Hoyle, C.E.; Hensel, R.D. \& Grubb, M.B. - J. Polym. Sci., Polym. Chem. Ed., 22, p.1865, (1984).

40 Hoyle, C.E.; Hensel, R.D. \& Grubb, M.B. - J. Radiat. Curing, 11, p.22, (1984).

41 Hoyle, C.E.; Hensel, R.D. \& Grubb, M.B. - Polym. Photochem., 4, p.69, (1984).

42 Haigh, J.; Brookes, A; Hendra, P.J.; Strawn, A; Nicholas, C. \& Purbrick, M. - Spectrochim. Acta A, 53, p.9, (1997).

43 Özpozan, T.; Schrader, B. \& Keller, S. - Spectrochim. Acta A, 53, p.1, (1997).
44 Rust, J.B.; Miller, L.J. \& Margerum, J.D. - Polym. Eng. Sci., 9, p.40, (1969).

45 Ottaviani, M.F.; Fioini, A.; Mason, P.N.; Corvaja, C. - Dent. Mater., 8, p.118, (1992).

46 Burtscher, P. - Dent. Mater. 8, p.218, (1993).

47 Rabek, J.K. - "Experimental Methods in Photochemistry and Photophysics", John Wiley, New York, (1982).

48 Damoun, S.; Papin, R.; Ripault, G.; Rousseau, M.; Rabadeaux, J. C.; Duran, D. - J. Raman Spectros., 23, p.385, (1992).

49 Witke, K.\& Kimmer, W.- Plaste and Kautschuk, 23, p.799, (1976).

50 Buchviser, S.F.; Gehlen, M.H. - J. Chem. Soc. Faraday Trans., 93, p.1133, (1997).

51 Mora, J.E.; Schroeter, S.R. \& Sutz, A.R. - Org. Coat. Plast. Preprints, 35, p.239, (1975).

52 Sastre, R.; Conde, M. \& Mateo, J.L. - J. Photochem. Photobiol., A Chem., 44, p.111, (1988).

53 Sastre, R.; Conde, M.; Catalina, F. \& Mateo, J.L. - Rev. Plást. Mod., 934, p.375, (1989).

54 Costa, M.L.; Rezende, M.C.; Pardini, L.C. - Quim. Nova, 23, p.320, (2000).

55 De Mello, C.B. \& Sano, W. - Anais $12^{\circ}$ CBECIMAT, 3, p.1226, (1996).

56 Ferreira da Silva, A.; Souza da Silva, T.; Nakamura, O.; d'Aguiar Neto, M.M.F.; Pepe, I.; Roman, L.S.; Veje, E. - Mater. Res., 4, p.23, (2001).

57 Sanchez, R.R.; Rieumont, J.B.; Cardoso, S.L.; da Silva, M.G.; Sthel, M.S.; Massunaga, M.S.O.; Gatts, C.N.; Vargas, H. - J. Braz. Chem. Soc., 10, p.97(1999).

Recebido: 20/08/03 Aprovado: 10/10/03 